\title{
Distinguishability and identifiability testing of contact state models
}

\author{
THOMAS J. DEBUS ${ }^{1, *}$, PIERRE E. DUPONT ${ }^{1}$ and ROBERT D. HOWE ${ }^{2}$ \\ ${ }^{1}$ Department of Aerospace and Mechanical Engineering, Boston University, Boston, \\ MA 02215, USA \\ ${ }^{2}$ Division of Engineering and Applied Sciences, Harvard University, Cambridge, MA 02138, USA
}

Received 11 August 2004; accepted 19 October 2004

\begin{abstract}
An important component of compliant motion control is the estimation of contact states during task execution. This paper addresses two fundamental questions that must be answered when formulating mathematical descriptions of contact states: are the contact states distinguishable from each other? and can the unknown or imprecisely known parameters in these descriptions be identified? An analytical method is presented for evaluating the distinguishability and identifiability questions of a set of contact state models described by non-linear algebraic equations. In contrast to existing, on-line numerical techniques, this approach can be used during the design phase of a contact state estimator to select contact models and robot sensors to ensure feasibility of the estimator. The approach is illustrated using contacts between polygonal and polyhedral objects.
\end{abstract}

Keywords: Distinguishability; identifiability; contact state estimation; polyhedra.

\section{INTRODUCTION}

The control of contact between manipulated objects is fundamental to a broad variety of robotic tasks. In fact, many tasks can be decomposed into a sequence of contact states between objects. At each step of task execution, motion planning and control involve moving from one contact state to another. In these situations, the robot must be able to perceive and distinguish between all of the contact states involved in task execution. Furthermore, in order to implement contact-based motion planning and control laws, it is necessary to estimate during contact the parameters describing the contact.

Contact-state estimators attempt to solve both of these problems using sensor data collected during task execution [1-4]. Contact state models are usually based on kinematic closure or wrench and twist constraints.

\footnotetext{
*To whom correspondence should be addressed. E-mail: tdebus@bu.edu
} 
The implementation of contact-state estimators requires local numerical tools based on sensor data to estimate unknown model parameters [2, 5] and to detect active contact states [1-4]. In contrast, the design of contact-state estimators require global analytical tools that can ensure, before implementation, that the proposed set of contact state models and associated sensors are sufficient (1) to distinguish each contact state from the others in the task and (2) for each contact state, to identify the contact model's parameters needed for motion planning and control. This paper focuses on the estimator design problem, proposing global analytical techniques for assessing the distinguishability and identifiability of contact state models.

Several analytical approaches based on geometry and/or force have been developed to test distinguishability [6-9]. In particular, the concept of contact equivalence presented by Xiao and Zhang [9] is based on analytical derivations of the equations describing the relative motions between contacting polygonal objects. This concept is used to characterize contact states that have equivalent structural equations (i.e., they are indistinguishable). Another example is Rosell et al. [7], in which tools investigating the robustness of paths generated by gross-motion planners to model uncertainty are presented. In particular, the distinguishability of potential contact situations due to uncertainty is analyzed using generalized force domains.

While distinguishability has to do with discriminating one contact state from another based on sensor data, identifiability addresses the questions of what parameters in a particular contact state's model can be estimated and, if so, how many solutions are possible. In the robot calibration literature, an identifiable model corresponds to a minimal parameterization [10]. In this literature, identifiability has been mostly addressed with numerical techniques [5, 11]. For example, analysis of the Jacobian matrix singular values [11, 12] or condition number [5] can be used as local tests for identifiability. A model is unidentifiable if the parameter Jacobian is singular (i.e., high condition number) and a model is locally identifiable if the Jacobian is far from a singularity (i.e., a condition number less than 100 [5]). An exception to numerical techniques is the work of Gautier and Khalil [10] in which symbolic computations extract the minimum set of inertial parameters used to represent the dynamic model of serial robots. The only analytical result related to contact identifiability known to the authors is the notion of C-space equivalence defined by Eberman in Ref. [13]. This technique can be applied only if the contact model can be written as a linear function of the sensor variables. To prove identifiability, Eberman's approach was to demonstrate the uniqueness of the mapping between the coefficients of the sensor variables (typically non-linear functions of the parameters) and the parameter values.

In contrast with the analytical techniques proposed in the literature that focus either on distinguishability or identifiability, the proposed approach presents a unified technique for the testing of both properties. Moreover, it can be applied to any contact model that can be written as homogeneous equations, regardless of the sensing modality (e.g., pose, wrench, twist) and dimensionality (e.g., planar, spatial) chosen to represent the model. 
Note the number of tests needed to assess distinguishability and identifiability of contact state models for a given task is independent of the method used for testing. In particular, for a task with $n$ contact states (where $n$ includes any possible multiple contacts), identifiability must be tested for each contact model and pairwise distinguishability testing requires additional $C_{2}^{n}$ tests. Given assumptions on geometry (e.g, polyhedral objects) and contact equations (e.g., twist), large classes of tasks can be composed from a set of principal contacts. Distinguishability and identifiablity testing of this principal contact set need be done only once to establish these properties for all tasks composed from this model set.

The concept of analytical testing of model distinguishability and identifiability is well established in the state space model literature with applications related to control [14], biology [15] and chemistry [16]. Distinguishability has been treated in Refs [16, 17] while identifiability has been examined in Refs [14, 15, 18].

In a series of papers published in the mid-eighties $[16,18,19]$ Walter and cowokers provided a uniform approach to testing the distinguishability and identifiability of state-space models. Using models $M_{i}(p)$ as given in (1) in which $X$ is the state vector $p$ is a set of unknown time-independent parameters, $U$ is the input vector and $Y$ is the output vector, Walter and co-workers defined distinguishability and identifiability as follows.

$$
M_{i}(p)=\left\{\begin{array}{l}
\dot{X}(t)=f(X(t), U(t), p, t) \\
Y_{i}(t, p)=g(X(t), p, t),
\end{array} \quad X(0)=X_{0}, U(0)=U_{0} .\right.
$$

Two state-space models, $M_{1}(p)$ and $M_{2}(q)$, are distinguishable if (i) for almost any $q$ there is no $p$ such that $Y_{1}(t, p)=Y_{2}(t, q)$ and (ii) for almost any $p$ there is no $q$ such that $Y_{2}(t, q)=Y_{1}(t, p)$, for any input and time [20]. Similarly, a statespace model $M(p)$ is globally (locally) identifiable if for almost any $q$ there is only one (a finite number of) $p$ such that $Y(t, p)=Y(t, q)$ for any input and time [20].

Given these definitions, there exist a variety of techniques to solve for state space model distinguishability and identifiability. For linear models, these methods include equating transfer function coefficients [19] and similarity transformations [21]. For non-linear models, techniques include linearization [22], Taylor series expansions [23] and generating series [20].

In this paper, it is shown that Walter and Lecourtier's definitions of distinguishability and identifiability can be adapted to any contact state models described by homogeneous non-linear algebraic equality constraints. Building on our previous work [24], a Taylor series approach is developed to analytically test distinguishability and identifiability of algebraic models which are non-linear in both the parameters and sensor values. The method can be used to select sensors and models during the design phase of a contact state estimator and is independent of the estimation technique selected for implementation.

The fact that the two notions of model identifiability and distinguishability have been investigated in several fields under different names can lead to confusion and misinterpretation. In this paper, the notion of contact distinguishability is equivalent 
to the notions of contact recognizability $[6,8]$ or contact identifiability $[7,25]$ presented in the motion planning literature. It is important to note that the concept of model identifiability presented in this paper refers to the identifiability of the parameters used to describe the model structure and not to the identification of the contact model. These choices of notations are inspired by the well established nomenclature presented in the state-space model literature [14-23].

In the next section, assumptions on the contact models are presented and the concepts of distinguishability and identifiability are presented in the context of these models. Section 3 presents the proposed technique for testing distinguishability and identifiability. Examples are provided in Section 4 followed by a complexity analysis of the method. Conclusions are presented in the Section 5 of the paper.

\section{DISTINGUISHABILITY AND IDENTIFIABILITY OF CONTACT MODELS}

In the literature, a variety of representations have been used to describe contact states; the two main approaches involve either a geometric description [2,9] or a force description [1, 6, 25] of the contact's kinematic constraints. Only the geometric contact model description is considered in this paper. Without loss of generality, contact models are considered for pairs of objects. One object, called the manipulated object, is assumed to be gripped and manipulated by a robot. The second object is called the environment object. The following assumptions are also assumed to apply.

- Objects are rigid polygons or polyhedrons.

- The manipulated object does not slip in the gripper.

- The environment object is fixed with respect to a world coordinate frame.

- In the most general case, the configuration (position and orientation) of the manipulated object with respect to the gripper is unknown and the configuration of the environment object with respect to a world frame is unknown. The parameters associated with the objects' configurations ( 6 for polygonal models and 12 for polyhedral models) constitute unknown parameters in the contact models.

- Contact models are comprised of non-linear equalities involving configuration parameters and sensor variables. Inequality constraints (e.g., overlap constraint and non-penetration constraint [9]) are not considered.

- Uncertainty in sensor variables (noise) is not considered.

\subsection{Motivating example}

To introduce formal definitions of contact model distinguishability and identifiability, it is worthwhile to first present simple examples of contact state models. Figure 1 depicts two polygonal contact states which can occur during planar peg insertion. Contact state 1 corresponds to contact between a vertex of the manipulated polygon and an edge of the environment polygon (i.e., shaded block). Contact state 2 


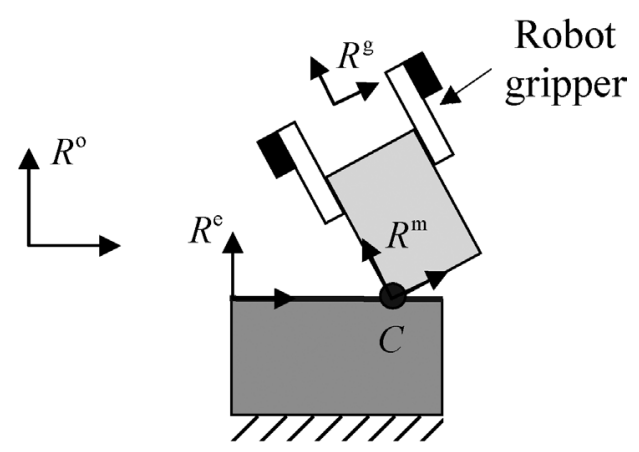

(a)

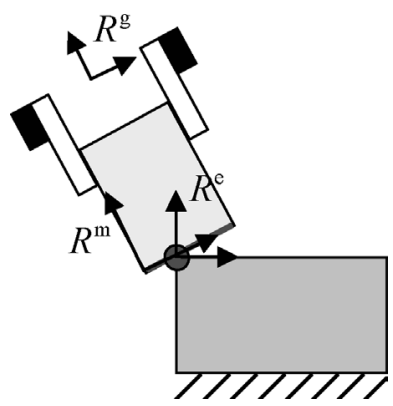

(b)

Figure 1. (a) Contact state A and (b) Contact state B.

describes contact between an edge of the manipulated polygon and a vertex of the environment polygon.

$R^{\mathrm{o}}, R^{\mathrm{g}}, R^{\mathrm{m}}$ and $R^{\mathrm{e}}$ represent coordinate frames for the world, the gripper, the manipulated object and the environment object, respectively.

Using the method described in Ref. [2], the constraints imposed by the contact states can be expressed in the world frame using kinematic closure equations:

$$
\vec{\varepsilon}^{\mathrm{o}}=T_{\mathrm{o}}^{\mathrm{g}}(t) T_{\mathrm{g}}^{\mathrm{m}} C^{\mathrm{m}}-T_{\mathrm{o}}^{\mathrm{e}} C^{\mathrm{e}} .
$$

Here, $\vec{\varepsilon}^{0}$ is the residual error vector between the contact point on the manipulated object and the contact point on the environment object. $T_{\mathrm{o}}^{\mathrm{g}}(t)$ is a homogeneous transform matrix relating the gripper frame to the world frame which is a function of the robot's sensor variables, e.g., joint angles. $T_{\mathrm{g}}^{\mathrm{m}}$ is a function of the unknown, but constant parameters describing the configuration of the manipulated object to the gripper frame. Similarly, $T_{\mathrm{o}}^{\mathrm{e}}$ describes the fixed configuration of the environment object in the world frame using unknown, constant parameters. The position of the contact point is represented by $C^{\mathrm{m}}$ in the frame associated with the manipulated object and by $C^{\mathrm{e}}$ in the frame associated with the environment object. These positions are expressed using unknown geometric parameters (e.g., vertex location, edge orientation) and time-dependent unknowns.

To eliminate the time-dependent parameters, the residual equation (2) is projected orthogonally to the direction of the time-varying contact coordinates. In Fig. 1, the orthogonal direction corresponds to the edge normal for both edge-vertex contacts. The result of this projection is a scalar, $\varepsilon_{\mathrm{p}}$, corresponding to an interpenetration distance, as given by (3). Its sign indicates either the interpenetration of the two objects or the distance between them.

$$
\varepsilon_{\mathrm{p}}=\vec{\varepsilon} \cdot \vec{n}
$$

The interpenetration distance, $\varepsilon_{\mathrm{p}}$, can be used as a residual in an implementation of an estimator as described in Ref. [2]. For the purpose of testing distinguishability 
and identifiability, the interpenetration distance is taken to be zero resulting in the following models for the contact states depicted in Fig. 1.

Contact State A

$$
\begin{aligned}
\varepsilon_{\mathrm{p}}^{\mathrm{a}}=0= & p_{5}+\left(p_{2} p_{3}-p_{1} p_{4}\right) \cos \theta(t)-\left(p_{1} p_{3}+p_{2} p_{4}\right) \sin \theta(t) \\
& +p_{2} x(t)-p_{1} y(t), \\
p_{1}^{2}+p_{2}^{2}= & 1 .
\end{aligned}
$$

Contact State B

$$
\begin{aligned}
\varepsilon_{\mathrm{p}}^{\mathrm{b}}=0= & q_{5}+\left(q_{1} q_{4}-q_{2} q_{3}+q_{2} x(t)-q_{1} y(t)\right) \cos \theta(t) \\
& -\left(q_{1} q_{3}+q_{2} q_{4}-q_{1} x(t)-q_{2} y(t)\right) \sin \theta(t), \\
q_{1}^{2}+q_{2}^{2}= & 1 .
\end{aligned}
$$

The parameters $\left[p_{3}, p_{4}\right]$ and $\left[q_{3}, q_{4}\right]$ represent the origin of the frames $R^{\mathrm{m}}$ and $R^{\mathrm{e}}$ with respect to the frames $R^{\mathrm{g}}$ and $R^{\mathrm{o}}$, respectively. Similarly, the parameter pairs $\left[p_{1}, p_{2}\right]^{\mathrm{T}}$ and $\left[q_{1}, q_{2}\right]^{\mathrm{T}}$ are the cosine and sine pairs of the angles describing the orientation of the frames $R^{\mathrm{e}}$ and $R^{\mathrm{m}}$ with respect to the frames $R^{\mathrm{o}}$ and $R^{\mathrm{g}}$. The parameter $p_{5}$ represents the magnitude of the position vector between the world frame and the environment frame projected along the normal of the contact's edge. Similarly, the parameter $q_{5}$ represents the magnitude of the position vector between the gripper frame and the manipulating frame projected along the normal of the contact's edge.

To estimate the parameters $p$ and $q$ in these equations, a sensor path $s(t), t \in$ $\left\{t_{0}, t_{1}, \ldots, t_{n}\right\}$, consisting of a discrete, finite set of positions and orientations of the robot gripper (i.e., $\left.s(t)=\left\{s_{1}(t), s_{2}(t), s_{3}(t)\right\}=\{x(t), y(t), \theta(t)\}\right)$ is needed. The minimum value of path length $n$ is determined by the number of unknown parameters and the nature of the constraint equations. The sets $p=\left\{p_{1}, \ldots, p_{5}\right\}$ and $q=\left\{q_{1}, \ldots q_{5}\right\}$ comprise the unknown time-independent parameters in the constraint equations.

Given non-linear algebraic models of contacts states, parameterized by sensor variables, $s(t)$, and time-independent configuration parameters, $p$, (e.g., model (4) for contact state A) and $q$ (e.g., model (5) for contact state B), distinguishability and identifiability can be defined in the manner of Walter and Lecourtier [20] as follows.

Definition 1. Two contact state models $\mathrm{A}$ and $\mathrm{B}$, parameterized by a sensor path $s(t)$ and by configuration parameters ( $p$ for A and $q$ for B) are distinguishable if, for almost any sensor path $s(t)$ of sufficient length, there is no solution for the parameter set $\{p, q\}$ such that the contact models are satisfied simultaneously.

DEFINITION 2. Contact model A (respectively, model B) is globally identifiable if, given almost any sensor path $s(t)$ of sufficient length, there exists a unique solution for $p$ (respectively, $q$ ) that satisfies model A (respectively, model B). If 
there are a finite number of solutions then contact model A (respectively, B) is locally identifiable.

In these definitions, the sensor path must be at least of the minimum length $n$. Also, note that while the path configurations need not be ordered or even correspond to contiguous configurations, they do need to satisfy excitation conditions. The phrase, 'almost any sensor path' is meant to rule out unexciting paths. In Definition 2, local identifiability is equivalent to the model being minimal (i.e., a model in which unidentifiable parameters are eliminated or grouped into terms which have a finite number of solutions [10]).

The following section develops a systematic method for evaluating these definitions for contact models of the form given by (4) and (5). The method is applied to these models and to others in Section 4.

\section{TAYLOR SERIES TESTING OF DISTINGUISHABILITY AND IDENTIFIABILITY FOR CONTACT STATES}

The testing of distinguishability and identifiability is based on finding practical ways to analyze and compare equations. In that regard, Taylor series expansion can be effective since it allows a non-linear model to be written as a unique set of algebraic equations in which each equation corresponds to a coefficient of the series. Thus, Taylor series reduce the distinguishability and identifiability testing to a comparison of the different coefficients of the series. This technique was successfully applied to testing the identifiability and distinguishability of zero-input state-space models [20, 23]. In this approach, the Taylor series of the output vector is written as a succession of time derivatives evaluated at time $t=0^{+}$given that the functions $f$ and $g$ and the vectors $X$ and $U$ in (1) are infinitely differentiable with respect to time. These coefficients form a set of algebraic equations that must be solved for all the possible sets of parameters. Note that these sets of equations can be difficult to solve by hand; however, tools from commutative algebra can be used to simplify the equations [17].

In developing a Taylor series approach for contact models, the model $M$ is permitted to be non-linear in the parameters $p$ as well as the sensor variables $s(t)$,

$$
M:\left\{\begin{array}{l}
F(p, s(t))=0 \\
H(p)=0 .
\end{array}\right.
$$

$F(\cdot)$ includes all the sensor-dependent equality constraints while $H(\cdot)$ models any additional equality constraint on the parameters (e.g., $H(p)=p_{1}^{2}+p_{2}^{2}-1$ in (4)). 
Assuming that the function $F(p, s)$ is analytic, a Taylor series expansion of order $k$ with respect to the sensor variables can be written as:

$$
\left\{\begin{array}{l}
F(p, s)=\sum_{i=0}^{k} a_{i}\left(p, s_{0}\right) \frac{\left(s-s_{0}\right)^{i}}{k !} \\
a_{i}\left(p, s_{0}\right)=\left.\frac{d^{i} F(p, s)}{d s^{i}}\right|_{s=s_{0}} .
\end{array}\right.
$$

Note that each coefficient of the Taylor series (i.e., $\left.a_{i}\left(p, s_{0}\right)\right)$ is a function of the unknown constant parameters and the known sensor values. If more than one sensor variable appears in the model, then partial derivatives with respect to all the sensor variables can be computed. Equation (8) shows a second-order expansion of a function of three variables $\left\{s_{1}, s_{2}, s_{3}\right\}$. This expansion could be applied to the examples presented in (4) and (5) by substituting $\left\{s_{1}, s_{2}, s_{3}\right\}$ by $\{x, y, \theta\}$.

$$
\left\{\begin{array}{l}
F(p,\{x, y, \theta\})=\left.F(p, s)\right|_{s=s_{0}}+\left.\sum_{i=1}^{3} \frac{\partial F(p, s)}{\partial s_{i}}\right|_{s=s_{0}}\left(s_{i}-s_{i 0}\right) \\
\quad+\left.\sum_{i=1}^{3} \sum_{j=1}^{3} \frac{\partial^{2} F(p, s)}{\partial s_{i} \partial s_{j}}\right|_{s=s_{0}} \frac{\left(s_{i}-s_{i 0}\right)\left(s_{j}-s_{j 0}\right)}{2 !}+\cdots \\
s=\left\{s_{1}, s_{2}, s_{3}\right\} \\
s_{0}=\left\{s_{10}, s_{20}, s_{30}\right\} .
\end{array}\right.
$$

Since the function $F(p, s)$ is assumed to be infinitely differentiable with respect to its sensor variables, its mixed derivatives are equal. With $m$ as the number of sensor variables and $k$ as the order of the expansion, the number of coefficients $n_{c}$ of the series is given by (9).

$$
n_{c}=\frac{(k+m) !}{k ! m !}
$$

\subsection{Distinguishability}

Based on the uniqueness of the Taylor series expansion, two contact state models are equivalent if and only if all the coefficients of their expansions are equal, as given below.

$$
A(p, s)=B(q, s) \Leftrightarrow\left\{\begin{array}{c}
a_{0}\left(p, s_{0}\right)=b_{0}\left(q, s_{0}\right) \\
a_{1}\left(p, s_{0}\right)=b_{1}\left(q, s_{0}\right) \\
\vdots \\
a_{n}\left(p, s_{0}\right)=b_{n}\left(q, s_{0}\right) \\
H_{1}(p)=H_{2}(q) .
\end{array}\right.
$$

This equality leads to the following test for distinguishability.

Definition 3. Two contact state models A and B in the form of (6) are distinguishable if and only if for any $s_{0}$, (i) given any choice of $p$, there is no 
solution to (10) for $q$, and (ii) given any choice of $q$, there is no solution to (10) for $p$.

To demonstrate that two models $\mathrm{A}$ and $\mathrm{B}$ are distinguishable, their Taylor coefficients (i.e., $a\left(p, s_{0}\right)$ and $\left.b\left(q, s_{0}\right)\right)$ must differ in at least one equation of (10) for all choices of parameters.

\subsection{Identifiability}

The identifiability of a contact model can be tested by considering how many sets of parameters yield the same Taylor series coefficients in (7). This test can be performed by counting the number of solutions for $p$, given $\hat{p}$, in the equation below.

$$
A(p, s)=A(\hat{p}, s) \Leftrightarrow\left\{\begin{array}{c}
a_{0}\left(p, s_{0}\right)=a_{0}\left(\hat{p}, s_{0}\right) \\
a_{1}\left(p, s_{0}\right)=a_{1}\left(\hat{p}, s_{0}\right) \\
\vdots \\
a_{n}\left(p, s_{0}\right)=a_{n}\left(\hat{p}, s_{0}\right) \\
H_{1}(p)=H_{1}(\hat{p}) .
\end{array}\right.
$$

This test can be stated formally as follows.

DEFINITION 4. A contact state model A is identifiable if and only if, given any $\hat{p}$ and any $s_{0}$, there is a unique solution to (11), which is $p=\hat{p}$. If a finite number of solutions for $p$ exist then $\mathrm{A}$ is locally identifiable. A is unidentifiable if an infinite number of solutions exist.

Since the Taylor series is developed around nominal sensor values, the approach appears to be local in the space of sensor variables. It is important to note, however, that the solutions for the parameters are obtained without substituting numerical values for the sensor values $s_{0}$ and so the results are truly global in the sensor space. To test identifiability involves solving for the number of solutions for $p$ in (11). Note that the solution $p=\hat{p}$ always exists.

\section{EXAMPLES}

Four examples are presented to illustrate the Taylor series technique of testing the distinguishability and identifiability of contact models for polygonal and polyhedral objects. The first two test the distinguishability and identifiability of a pair of polygonal contact models, while the last two focus on the identifiability and distinguishability testing of a pair of polyhedral contact models.

\subsection{Example 1: distinguishability of polygonal vertex-edge contact models}

As a first example, the distinguishability of the models developed for the contact states of Fig. 1 is tested. The models given by (4) and (5) can be written in the form 
of (6) as follows:

$$
\begin{aligned}
\mathrm{A}: & F^{\mathrm{a}}(p, s)=p_{5}+\left(p_{2} p_{3}-p_{1} p_{4}\right) \cos \theta(t)-\left(p_{1} p_{3}+p_{2} p_{4}\right) \sin \theta(t) \\
& +p_{2} x(t)-p_{1} y(t)=0 \\
& H^{\mathrm{a}}(p)=p_{1}^{2}+p_{2}^{2}-1=0, \\
\mathrm{~B}: & F^{\mathrm{b}}(q, s)=q_{5}+\left(q_{1} q_{4}-q_{2} q_{3}+q_{2} x(t)-q_{1} y(t)\right) \cos \theta(t) \\
& -\left(q_{1} q_{3}+q_{2} q_{4}-q_{1} x(t)-q_{2} y(t)\right) \sin \theta(t)=0 \\
& H^{\mathrm{b}}(q)=q_{1}^{2}+q_{2}^{2}-1=0 .
\end{aligned}
$$

Series coefficients are given in (14) for contact A and in (15) for contact B. Due to the cyclic nature of the derivatives of sine and cosine, derivative terms beyond second order do not generate independent equations.

$$
\left\{\begin{aligned}
& a_{0}= F^{\mathrm{a}}\left(p, s_{0}\right)=p_{5}+\left(p_{2} p_{3}-p_{1} p_{4}\right) \cos \theta_{0}-\left(p_{1} p_{3}+p_{2} p_{4}\right) \sin \theta_{0} \\
& \quad+p_{2} x_{0}-p_{1} y_{0}=0 \\
& a_{1}= F_{\theta}^{\mathrm{a}}\left(p, s_{0}\right)=-\left(p_{1} p_{3}+p_{2} p_{4}\right) \cos \theta_{0}-\left(p_{2} p_{3}-p_{1} p_{4}\right) \sin \theta_{0} \\
& a_{2}= F_{x}^{\mathrm{a}}\left(p, s_{0}\right)=p_{2} \\
& a_{3}= F_{y}^{\mathrm{a}}\left(p, s_{0}\right)=-p_{1} \\
& a_{4}= F_{\theta \theta}^{\mathrm{a}}\left(p, s_{0}\right)=-\left(p_{2} p_{3}-p_{1} p_{4}\right) \cos \theta_{0}+\left(p_{1} p_{3}+p_{2} p_{4}\right) \sin \theta_{0} \\
& a_{5}=F_{\theta x}^{\mathrm{a}}\left(p, s_{0}\right)=0 \\
& a_{6}=F_{\theta y}^{\mathrm{a}}\left(p, s_{0}\right)=0 \\
& a_{7}=F_{x x}^{\mathrm{a}}\left(p, s_{0}\right)=0 \\
& a_{8}=F_{x y}^{\mathrm{a}}\left(p, s_{0}\right)=0 \\
& a_{9}=F_{y y}^{\mathrm{a}}\left(p, s_{0}\right)=0
\end{aligned}\right.
$$

$$
\left\{\begin{aligned}
b_{0}= & F^{\mathrm{b}}\left(q, s_{0}\right)=q_{5}+\left(q_{1} q_{4}-q_{2} q_{3}+q_{2} x_{0}-q_{1} y_{0}\right) \cos \theta_{0} \\
& -\left(q_{1} q_{3}+q_{2} q_{4}-q_{1} x_{0}-q_{2} y_{0}\right) \sin \theta_{0}=0 \\
b_{1}= & F_{\theta}^{\mathrm{b}}\left(q, s_{0}\right)=-\left(q_{1} q_{3}+q_{2} q_{4}-q_{1} x_{0}-q_{2} y_{0}\right) \cos \theta_{0} \\
& -\left(q_{1} q_{4}-q_{2} q_{3}+q_{2} x_{0}-q_{1} y_{0}\right) \sin \theta_{0} \\
b_{2}= & F_{x}^{\mathrm{b}}\left(q, s_{0}\right)=q_{2} \cos \theta_{0}+q_{1} \sin \theta_{0} \\
b_{3}= & F_{y}^{\mathrm{b}}\left(q, s_{0}\right)=-q_{1} \cos \theta_{0}+q_{2} \sin \theta_{0} \\
b_{4}= & F_{\theta \theta}^{\mathrm{b}}\left(q, s_{0}\right)=-\left(q_{1} q_{4}-q_{2} q_{3}+q_{2} x_{0}-q_{1} y_{0}\right) \cos \theta_{0} \\
& +\left(q_{1} q_{3}+q_{2} q_{4}-q_{1} x_{0}-q_{2} y_{0}\right) \sin \theta_{0} \\
b_{5}= & F_{\theta x}^{\mathrm{b}}\left(q, s_{0}\right)=-q_{2} \sin \theta_{0}+q_{1} \cos \theta_{0} \\
b_{6}= & F_{\theta y}^{\mathrm{b}}\left(q, s_{0}\right)=q_{1} \sin \theta_{0}+q_{2} \cos \theta_{0} \\
b_{7}= & F_{x x}^{\mathrm{b}}\left(q, s_{0}\right)=0 \\
b_{8}= & F_{x y}^{\mathrm{b}}\left(q, s_{0}\right)=0 \\
b_{9}= & F_{y y}^{\mathrm{b}}\left(q, s_{0}\right)=0
\end{aligned}\right.
$$

Applying Definition 3 to test the distinguishability of models (12) and (13) involves combining (12)-(15) in the form of (10). It can be directly observed that the pair of 
equations below cannot be satisfied since they contradict $q_{1}^{2}+q_{2}^{2}=1$.

$$
\left\{\begin{array} { l } 
{ a _ { 5 } = b _ { 5 } } \\
{ a _ { 6 } = b _ { 6 } }
\end{array} \Leftrightarrow \left\{\begin{array}{l}
0=-q_{2} \sin \theta_{0}+q_{1} \cos \theta_{0} \\
0=q_{1} \sin \theta_{0}+q_{2} \cos \theta_{0}
\end{array} \Rightarrow q_{1}^{2}+q_{2}^{2}=0 .\right.\right.
$$

Since the equations have no solution regardless of whether $p$ or $q$ is given, the two contact state models are distinguishable.

\subsection{Example 2: identifiability of a polygonal vertex-edge contact model}

The identifiability of the contact model representing the contact state of Fig. 1a is tested here. To apply Definition 4, at least five independent equations are needed to solve for the five parameters $p_{1}-p_{5}$. The Taylor series coefficients expressed in (14) provide five equations which can be combined with the last equation of (12) to obtain a set of six equations in the form of (11).

$$
\left\{\begin{array}{c}
p_{5}+\left(p_{2} p_{3}-p_{1} p_{4}\right) \cos \theta_{0}-\left(p_{1} p_{3}+p_{2} p_{4}\right) \sin \theta_{0}+p_{2} x_{0}-p_{1} y_{0} \\
=\hat{p}_{5}+\left(\hat{p}_{2} \hat{p}_{3}-\hat{p}_{1} \hat{p}_{4}\right) \cos \theta_{0}-\left(\hat{p}_{1} \hat{p}_{3}+\hat{p}_{2} \hat{p}_{4}\right) \sin \theta_{0}+\hat{p}_{2} x_{0}-\hat{p}_{1} y_{0}=0 \\
-\left(p_{1} p_{3}+p_{2} p_{4}\right) \cos \theta_{0}-\left(p_{2} p_{3}-p_{1} p_{4}\right) \sin \theta_{0}=-\left(\hat{p}_{1} \hat{p}_{3}+\hat{p}_{2} \hat{p}_{4}\right) \cos \theta_{0} \\
-\left(\hat{p}_{2} \hat{p}_{3}-\hat{p}_{1} \hat{p}_{4}\right) \sin \theta_{0} \\
p_{2}=\hat{p}_{2} \\
-p_{1}=-\hat{p}_{1} \\
-\left(p_{2} p_{3}-p_{1} p_{4}\right) \cos \theta_{0}+\left(p_{1} p_{3}+p_{2} p_{4}\right) \sin \theta_{0}=-\left(\hat{p}_{2} \hat{p}_{3}-\hat{p}_{1} \hat{p}_{4}\right) \cos \theta_{0} \\
+\left(\hat{p}_{1} \hat{p}_{3}+\hat{p}_{2} \hat{p}_{4}\right) \sin \theta_{0} \\
p_{1}^{2}+p_{2}^{2}= \\
\hat{p}_{1}^{2}+\hat{p}_{2}^{2}=1 .
\end{array}\right.
$$

To obtain all possible solutions for $p$ given $\hat{p}$, it can be seen that the third and fourth equations define uniquely $p_{1}$ and $p_{2}$ and that this solution also satisfies the sixth equation. Given $p_{1}$ and $p_{2}$, the remaining parameters appear linearly in the three remaining equations. Consequently, (17) admits only one solution, given below.

$$
\begin{aligned}
& p_{1}=\hat{p}_{1} \\
& p_{2}=\hat{p}_{2} \\
& p_{3}=\hat{p}_{3} \\
& p_{4}=\hat{p}_{4} \\
& p_{5}=\hat{p}_{5} .
\end{aligned}
$$

By Definition 4, contact states A is globally identifiable.

\subsection{Example 3: identifiability of a polyhedral vertex-face contact model}

To demonstrate the applicability of the approach to polyhedral models, this example considers the identifiability of the contact shown in Fig. 2. 


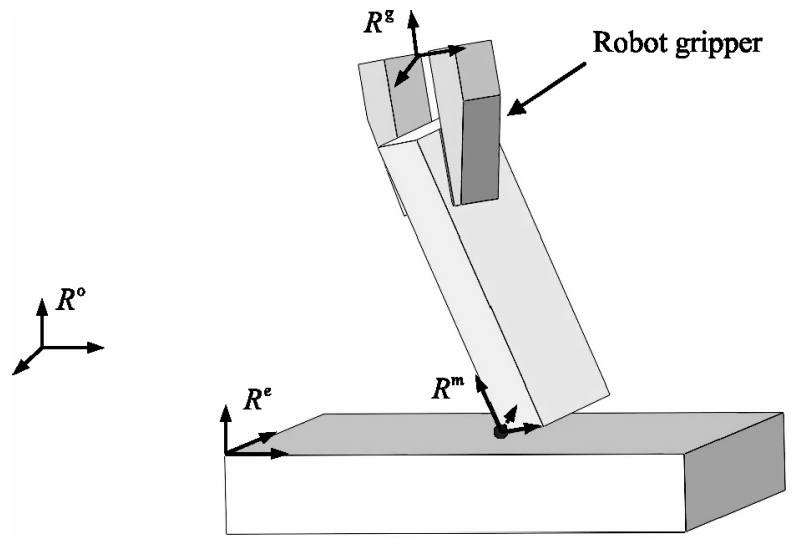

Figure 2. Contact state $\mathrm{C}$ : vertex-face contact state.

Using the techniques described in Section 2.1, the contact model can be written as follows:

$$
\mathrm{C}:\left\{\begin{array}{l}
F^{\mathrm{c}}(p, s)=p_{10}+K_{1} x(t)+K_{2} y(t)+K_{3} z(t) \\
+p_{7}\left[K_{1} \cos \beta(t) \cos \alpha(t)+K_{2} \cos \beta(t) \sin \alpha(t)-K_{3} \sin \beta(t)\right] \\
+p_{8}\left[\begin{array}{l}
K_{1}(\cos \alpha(t) \sin \gamma(t) \sin \beta(t)-\cos \gamma(t) \sin \alpha(t)) \\
+K_{2}(\cos \gamma(t) \cos \alpha(t)+\sin \gamma(t) \sin \beta(t) \sin \alpha(t)) \\
+K_{3} \cos \beta(t) \sin \gamma(t)
\end{array}\right] \\
+p_{9}\left[\begin{array}{l}
K_{1}(\cos \gamma(t) \cos \alpha(t) \sin \beta(t)+\sin \gamma(t) \sin \alpha(t)) \\
+K_{2}(-\cos \alpha(t) \sin \gamma(t)+\cos \gamma(t) \sin \beta(t) \sin \alpha(t)) \\
+K_{3} \cos \gamma(t) \cos \beta(t)
\end{array}\right]=0 \\
K_{1}=p_{1} p_{4} p_{5}+p_{2} p_{6} \\
K_{2}=p_{2} p_{4} p_{5}-p_{1} p_{6} \\
K_{3}=p_{3} p_{5} \\
p_{1}^{2}+p_{2}^{2}=1 \\
p_{3}^{2}+p_{4}^{2}=1 \\
p_{5}^{2}+p_{6}^{2}=1 .
\end{array}\right.
$$

Here, $\left[p_{1}, p_{2}, p_{3}, p_{4}, p_{5}, p_{6}\right]$ are used to parameterize the three unknown rotations used in $T_{\mathrm{o}}^{\mathrm{e}}$ and $\left[p_{7}, p_{8}, p_{9}\right]$ represent the unknown translations used in $T_{\mathrm{g}}^{\mathrm{m}}$. The parameter $p_{10}$ represents the magnitude of the position vector between the world and environment object frames projected on the normal of the contact's face. The sensor variables $\{x, y, z, \alpha, \beta, \gamma\}$ represent the known position and orientation of the robot gripper.

At least 10 equations are needed to solve for the ten parameters. Since 6 sensor variables are available, 7 Taylor coefficients are provided through first-order expansion. These equations can be combined with the last three equations of (19) to obtain the desired number of equations. Since $p_{1}-p_{6}$ are the only coefficients multiplying $\{x(t), y(t), z(t)\}$, the first-order coefficients with respect to these sens- 
ing variables can be combined with the last three constraint equations of (19) to produce the algebraic system of six equations,

$$
\left\{\begin{array}{l}
a_{1}=F_{x}^{\mathrm{c}}\left(p, s_{0}\right)=K_{1}=p_{1} p_{4} p_{5}+p_{2} p_{6} \\
a_{2}=F_{y}^{\mathrm{c}}\left(p, s_{0}\right)=K_{2}=p_{2} p_{4} p_{5}-p_{1} p_{6} \\
a_{3}=F_{z}^{\mathrm{c}}\left(p, s_{0}\right)=K_{3}=p_{3} p_{5} \\
p_{1}^{2}+p_{2}^{2}=1 \\
p_{3}^{2}+p_{4}^{2}=1 \\
p_{5}^{2}+p_{6}^{2}=1 .
\end{array}\right.
$$

This system admits an infinite number of solutions given in (21) with $p_{5}$ and $p_{6}$ as free parameters. Note that the choice of the free parameters is arbitrary; any pairs $\left\{p_{1}, p_{2}\right\},\left\{p_{3}, p_{4}\right\}$ or $\left\{p_{5}, p_{6}\right\}$ can be chosen to solve for the remaining parameters. By Definition 4, the contact state is unidentifiable.

$$
\left\{\begin{array}{l}
p_{1}=\frac{-2 \hat{p}_{2} \hat{p}_{4} p_{5} p_{6}+\hat{p}_{1}\left(p_{6}^{2}+\hat{p}_{4}^{2} p_{5}^{2}\right)}{p_{6}^{2}+\hat{p}_{4}^{2} p_{5}^{2}} \\
p_{2}=\frac{2 \hat{p}_{1} \hat{p}_{4} p_{5} p_{6}+\hat{p}_{2}\left(\hat{p}_{4}^{2} p_{5}^{2}+p_{5}^{2}-1\right)}{p_{6}^{2}+\hat{p}_{4}^{2} p_{5}^{2}} \\
p_{3}=\hat{p}_{3} \\
p_{4}=\hat{p}_{4} .
\end{array}\right.
$$

Equation (21) shows that two out of the three angles specifying the orientation of the environment object can be uniquely identified. This can be explained geometrically by noticing that the contact state is invariant under rotations of the environment object about the face's normal. This suggests that the contact should be re-parameterized using only two angles to model the orientation uncertainty of the environment object. In this case, $p_{5}$ and $p_{6}$ can be selected arbitrarily giving a unique solution to (21).

Note that over-parameterization is often a result of applying general modeling techniques, such as the example technique presented in Section 2.1. To obtain a minimal representation, i.e., at least locally identifiable model, the unidentifiable parameters must be grouped to form identifiable parameters or eliminated [10]. This reduction task can be difficult to implement analytically for arbitrary models. The proposed identifiability test is a general tool for detecting the presence of unidentifiable parameters.

Given that $p_{1}-p_{4}$ are identifiable, the identifiability of the remaining parameters can be investigated by looking at the four remaining first-order Taylor coefficients of (19), as shown in (22).

$$
\left\{\begin{array}{l}
a_{0}=F^{\mathrm{c}}\left(p, s_{0}\right)=p_{10}+K_{1} x_{0}+K_{2} y_{0}+K_{3} z_{0}+p_{7} U+p_{8} V+p_{9} W \\
a_{4}=F_{\alpha}^{\mathrm{c}}\left(p, s_{0}\right)=p_{7} U_{\alpha}+p_{8} V_{\alpha}+p_{9} W_{\alpha} \\
a_{5}=F_{\beta}^{\mathrm{c}}\left(p, s_{0}\right)=p_{7} U_{\beta}+p_{8} V_{\beta}+p_{9} W_{\beta} \\
a_{6}=F_{\gamma}^{\mathrm{c}}\left(p, s_{0}\right)=p_{7} U_{\gamma}+p_{8} V_{\gamma}+p_{9} W_{\gamma} .
\end{array}\right.
$$


In these equations, the variables $U, V, W$ and their derivatives are non-linear functions of the known variables $\alpha, \beta, \gamma, K_{1}, K_{2}$ and $K_{3}$. Moreover it can be shown that these four equations are linearly independent. As a consequence, $p_{7}-p_{10}$ can be uniquely identified, given that $K_{1}, K_{2}$ and $K_{3}$ are known. Therefore the vertexface contact model is said to be globally identifiable, as long as the orientation uncertainty of the environment object is parameterized by two angles.

\subsection{Example 4: distinguishability of polyhedral vertex-face contact models}

This example examines the distinguishability of two kinematically equivalent contact-state models in which a single vertex of the manipulated object is in contact with either of two orthogonal faces of the environment object as shown in Fig. 3. The contact equations characterizing the two models are presented in (23) and (24). Note that (23) and (24) are globally identifiable models obtained by selecting $p_{5}=q_{5}=1$ and $p_{6}=q_{6}=0$.

$$
\begin{aligned}
& \mathrm{C}^{\prime}:\left\{\begin{array}{l}
F^{\mathrm{c}^{\prime}}(p, s)=p_{10}+p_{1} p_{4} x(t)+p_{2} p_{4} y(t)+p_{3} z(t) \\
+p_{7}\left[p_{1} p_{4} \cos \beta(t) \cos \alpha(t)+p_{2} p_{4} \cos \beta(t) \sin \alpha(t)-p_{3} \sin \beta(t)\right] \\
+p_{8}\left[\begin{array}{l}
p_{1} p_{4}(\cos \alpha(t) \sin \gamma(t) \sin \beta(t)-\cos \gamma(t) \sin \alpha(t)) \\
+p_{2} p_{4}(\cos \gamma(t) \cos \alpha(t)+\sin \gamma(t) \sin \beta(t) \sin \alpha(t)) \\
+p_{3} \cos \beta(t) \sin \gamma(t)
\end{array}\right] \\
+p_{9}\left[\begin{array}{l}
p_{1} p_{4}(\cos \gamma(t) \cos \alpha(t) \sin \beta(t)+\sin \gamma(t) \sin \alpha(t)) \\
+p_{2} p_{4}(-\cos \alpha(t) \sin \gamma(t)+\cos \gamma(t) \sin \beta(t) \sin \alpha(t)) \\
+p_{3} \cos \gamma(t) \cos \beta(t)
\end{array}\right]=0 \\
p_{1}^{2}+p_{2}^{2}=1 \\
p_{3}^{2}+p_{4}^{2}=1,
\end{array}\right. \\
& \mathrm{D}:\left\{\begin{array}{l}
F^{\mathrm{d}}(q, s)=q_{10}+q_{1} q_{3} x(t)+q_{2} q_{3} y(t)-q_{4} z(t) \\
+q_{7}\left[q_{1} q_{3} \cos \beta(t) \cos \alpha(t)+q_{2} q_{3} \cos \beta(t) \sin \alpha(t)+q_{4} \sin \beta(t)\right] \\
+q_{8}\left[\begin{array}{l}
q_{1} q_{3}(\cos \alpha(t) \sin \gamma(t) \sin \beta(t)-\cos \gamma(t) \sin \alpha(t)) \\
+q_{2} q_{3}(\cos \gamma(t) \cos \alpha(t)+\sin \gamma(t) \sin \beta(t) \sin \alpha(t)) \\
-q_{4} \cos \beta(t) \sin \gamma(t)
\end{array}\right] \\
+q_{9}\left[\begin{array}{l}
q_{1} q_{3}(\cos \gamma(t) \cos \alpha(t) \sin \beta(t)+\sin \gamma(t) \sin \alpha(t)) \\
+q_{2} q_{3}(-\cos \alpha(t) \sin \gamma(t)+\cos \gamma(t) \sin \beta(t) \sin \alpha(t)) \\
-q_{4} \cos \gamma(t) \cos \beta(t)
\end{array}\right]=0 \\
q_{1}^{2}+q_{2}^{2}=1 \\
q_{3}^{2}+q_{4}^{2}=1 .
\end{array}\right.
\end{aligned}
$$

Since the orientation of the contacting face is the only difference between the two contacts, distinguishability is analyzed by looking at the parameters defining the unknown orientation of the two faces, i.e., $p_{1}-p_{4}$ for contact $\mathrm{C}^{\prime}$ and $q_{1}-q_{4}$ for contact D. As a consequence, the distinguishability problem reduces to the 

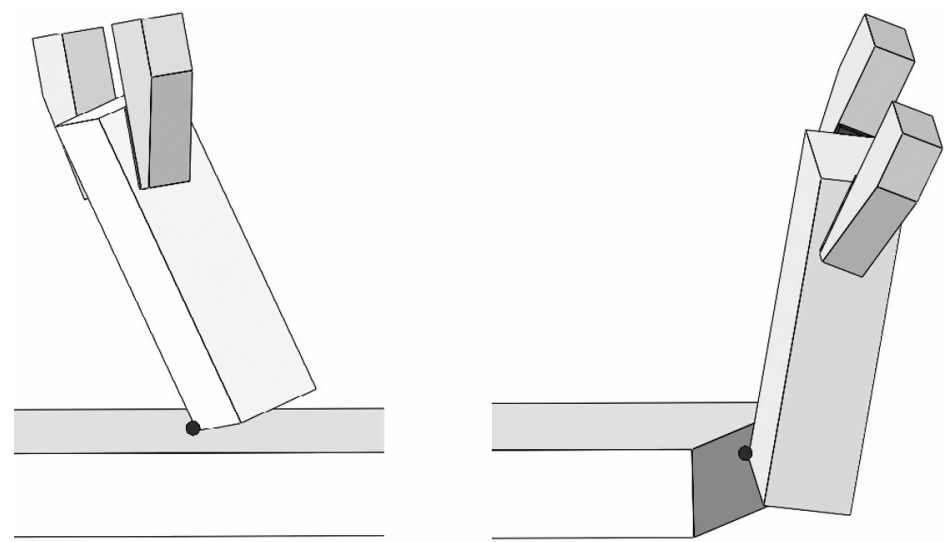

Figure 3. Two vertex-face contact states in which the location of the contact point is the same on the manipulated object but on two rthogonal faces of the environment object.

comparison of the first-order Taylor coefficients with respect to the position sensing variables. Definition 3 is applied to the equations formed by combining the three first-order coefficients of (23) and (24) together with the final equations of (23) and (24) in the form of (10).

$$
\begin{aligned}
\left\{\begin{array} { l } 
{ a _ { 1 } = b _ { 1 } } \\
{ a _ { 2 } = b _ { 2 } } \\
{ a _ { 3 } = b _ { 3 } } \\
{ p _ { 1 } ^ { 2 } + p _ { 2 } ^ { 2 } = q _ { 1 } ^ { 2 } + q _ { 2 } ^ { 2 } = 1 } \\
{ p _ { 3 } ^ { 2 } + p _ { 4 } ^ { 2 } = q _ { 3 } ^ { 2 } + q _ { 4 } ^ { 2 } = 1 }
\end{array} \Leftrightarrow \left\{\begin{array}{l}
F_{x}^{\mathrm{c}^{\prime}}\left(p, s_{0}\right)=F_{x}^{\mathrm{d}}\left(q, s_{0}\right) \\
F_{y}^{\mathrm{c}^{\prime}}\left(p, s_{0}\right)=F_{y}^{\mathrm{d}}\left(q, s_{0}\right) \\
F_{z}^{\mathrm{c}^{\prime}}\left(p, s_{0}\right)=F_{z}^{\mathrm{d}}\left(q, s_{0}\right) \\
p_{1}^{2}+p_{2}^{2}=q_{1}^{2}+q_{2}^{2}=1 \\
p_{3}^{2}+p_{4}^{2}=q_{3}^{2}+q_{4}^{2}=1
\end{array}\right.\right. \\
\Leftrightarrow\left\{\begin{array}{l}
p_{1} p_{4}=q_{1} q_{3} \\
p_{2} p_{4}=q_{2} q_{3} \\
p_{3}=-q_{4} \\
p_{1}^{2}+p_{2}^{2}=q_{1}^{2}+q_{2}^{2}=1 \\
p_{3}^{2}+p_{4}^{2}=q_{3}^{2}+q_{4}^{2}=1 .
\end{array}\right.
\end{aligned}
$$

The two solutions of (25) for $p$ given $q$ appear in (26). The same solutions arise when the system is solved for $q$ given $p$. These two solutions define the same orientation and, thus, constitute a single solution.

$$
\left\{\begin{array}{l}
p_{1}=q_{1} \\
p_{2}=q_{2} \\
p_{3}=-q_{4} \\
p_{4}=q_{3}
\end{array}, \quad\left\{\begin{array}{l}
p_{1}=-q_{1} \\
p_{2}=-q_{2} \\
p_{3}=-q_{4} \\
p_{4}=-q_{3}
\end{array}\right.\right.
$$


The final solution for (10) is given by (27).

$$
\left\{\begin{array}{l}
p_{1}=q_{1} \\
p_{2}=q_{2} \\
p_{3}=-q_{4} \\
p_{4}=q_{3} \\
p_{7}=q_{7} \\
p_{8}=q_{8} \\
p_{9}=q_{9} \\
p_{10}=q_{10} .
\end{array}\right.
$$

This solution yields the anticipated result indicating the orthogonality of the two contact faces. By Definition 3, since a solution exists, the two contact states are indistinguishable.

If, however, the orientation angle $\theta$ of the environment object with respect to the normal of its front face is known then

$$
\begin{aligned}
& p_{3}=q_{3}=\cos \theta \\
& p_{4}=q_{4}=\sin \theta,
\end{aligned}
$$

which contradicts (27) and so the contact states are distinguishable. This result can be explained geometrically by noticing that a $90^{\circ}$ rotation around the environment object's front face of the contact depicted in Fig. 3a leads to the contact pictured in Fig. 3b, making the two contacts indistinguishable. On the other hand, if the rotation angle is known, then the two contacts are distinguishable since there exists no transformation than can change Fig. 3a to Fig. $3 b$.

Recall that, in Example 3, the vertex-face contact model used here was made identifiable by eliminating the parameter corresponding to rotation about the face's normal. Example 4 reveals that distinguishability can further restrict the choice of parameterization (i.e., free parameters) above what is needed for identifiability.

\section{COMPUTATIONAL COMPLEXITY ANALYSIS}

The distinguishability and identifiability approaches presented in this paper reduce to solving the sets of algebraic equations in (10) and (11). Determining the actual number of solutions to sets of non-linear algebraic equations is a difficult challenge whose difficulty increases with the number of equations, $v$, in the set. In this section, upper and lower bounds, $v_{\min }$ and $v_{\max }$, are derived on the number of equations in the sets described by (10) and (11) for kinematic pose equations.

\subsection{Identifiability testing}

The complexity of the proposed approach depends on the size of the system of equations in (11) used to test identifiability. The goal of identifiability is 
to show that there is a unique set of parameters satisfying the system of nonlinear equations in (11). By construction, the system admits at least one solution. Since exactly determined systems of non-linear equations usually admit multiple solutions, however, additional equations may be needed to show uniqueness. As a consequence, a lower bound on the number of independent algebraic equations needed to solve for the unknowns is given by $n$, the number of unknown parameters associated with the given contact equation.

$$
v_{\min }=n \text {. }
$$

If $H(\cdot)$ in (11) provides $\beta$ equations, the number of Taylor series coefficient equations, $n_{c}$, needed to achieve this lower bound is given

$$
n_{c} \geqslant v_{\min }-\beta \text {. }
$$

This is a lower bound on $n_{c}$ since there is no guarantee that the algebraic equations from the Taylor series are independent. This bound on the number of coefficients can be related to the minimum order of the series, $k_{\min }$, needed to produce them by substituting the minimum value of $n_{c}$ from (30) into (9).

$$
k_{\min }=\min _{k=1,2,3, \ldots}\left[\frac{(k+m) !}{k ! m !}-v_{\min }+\beta\right]>0 .
$$

An upper bound on the series order can be derived by noting that kinematic pose equations involve only cyclic and polynomial functions of the sensor variables. As a consequence, the terms in the Taylor coefficients start repeating or go to zero after a finite number of differentiations. The number of differentiations required for a variable to repeat or go to zero is defined here as the degree of cyclicity of the variable and is denoted $C(\cdot)$.

The degree of cyclicity associated with the angular sensor variables $s_{\mathrm{a}}$ (e.g., $s_{\mathrm{a}}=\{\alpha(t), \beta(t), \gamma(t)\}$ in 3D) equals 4 , since, in every term (see, e.g., (19)) the angular variables appear as linear trigonometric functions, e.g., sine or cosine. The degree of cyclicity for the positional sensor variables $s_{\mathrm{p}}$ (e.g., $s_{\mathrm{p}}=\{x(t), y(t), z(t)\}$ in $3 \mathrm{D}$ ) equals 1 , since they appear linearly in the kinematic pose equations. See (19) as an example.

Since identifiability testing involves comparing sets of equations having identical structures (11), the signs of the repeating functions cancel, which reduces the cyclicity of the angular variables for identifiability testing from four to two:

$$
C\left(s_{\mathrm{a}}\right)=2, \quad C\left(s_{\mathrm{p}}\right)=1 .
$$

For example, when testing (15) for identifiability, it can be seen that $b_{3}$ and $b_{5}$ yield the same equations when written in the form of (11).

As a consequence, the upper bound $k_{\max }$ on the series order is given by

$$
k_{\max }=\operatorname{dim}\left(s_{\mathrm{a}}\right) \cdot C\left(s_{\mathrm{a}}\right),
$$


Table 1.

Bounds of the number of equations needed for identifiability testing

\begin{tabular}{llcccr}
\hline$v=n_{c}+\beta$ & $\beta$ & $v_{\min }$ & $v_{\max }^{*}$ & $v_{\max }$ & $v$ \\
\hline Example 2 & 1 & 5 & 6 & 11 & 5 \\
Example 3 & 3 & 10 & 33 & 927 & 10 \\
\hline
\end{tabular}

in which $\operatorname{dim}\left(s_{\mathrm{a}}\right)=1$ in two dimensions and $\operatorname{dim}\left(s_{\mathrm{a}}\right)=3$ in three dimensions. Therefore, the maximum number of equations available to test for identifiability is

$$
v_{\max }=\frac{\left(k_{\max }+m\right) !}{k_{\max } ! m !}+\beta .
$$

While $v_{\max }$ can be large, many of these equations are either zero or redundant. The subset $v_{\max }^{*} \leqslant v_{\max }$ of non-zero independent equations can be obtained using a computer algebra package.

The number of equations used to solve for identifiability is bounded as $v_{\min } \leqslant$ $v \leqslant v_{\max }^{*} \leqslant v_{\max }$. This bound can related to $n_{c}$, the number of Taylor coefficients, as

$$
v_{\min } \leqslant \beta+n_{c} \leqslant v_{\max }^{*} .
$$

Table 1 presents the lower and upper bounds as well as the actual number of equations needed to test for identifiability in Examples 2 and 3, corresponding to equations (12) and (19). In both cases, the actual number of coefficients needed corresponds to the lower bound.

\subsection{Distinguishability testing}

The goal of distinguishability testing is to show that there is no set of parameters satisfying the system of non-linear equations in (10). To do so, at least one contradiction, valid for all choices of parameters, must be found in these equations. Assuming that a contradiction is not present in $H_{1}(p)=H_{2}(q)$, at least one Taylor coefficient is needed to establish distinguishability, resulting in the following lower bound.

$$
n_{c} \geqslant 1 \Rightarrow v_{\min } \geqslant \beta+1 \text {. }
$$

In contrast to identifiability testing, the lack of structural similarities between the left and right sides of (10) prevent the simplifications presented for identifiability (i.e., elimination of the redundant and non-zero parameters). Therefore, an upper bound on the number of coefficients for distinguishability testing is not easily established. In practice, the Taylor coefficients are computed one by one until a contradiction is found. If no contradiction is found after a large number of coefficients (i.e., $k=10$ in (9)) then one gives up without drawing a conclusion on the distinguishability of the contact states. 
Table 2.

Bounds of the number of equations computed for distinguishability testing

\begin{tabular}{lllcr}
\hline$v=n_{c}+\beta$ & $\beta$ & $v_{\min }$ & $v_{\max }$ & $v$ \\
\hline Example 1 & 1 & 2 & 11 & 8 \\
Example 4 & 2 & 3 & 9 & 5 \\
\hline
\end{tabular}

Note that when a contradiction can be found, the actual number of equations computed for distinguishability testing depends on the order in which the Taylor coefficients are computed. In Example 1, the second-order mixed derivatives $F_{\theta x}$ and $F_{\theta y}$ are needed to prove distinguishability. These derivatives correspond to the sixth and seventh coefficients of (14) and (15), respectively. This choice in the order of the coefficients is arbitrary (e.g., $F_{\theta x}$ could be computed before $F_{\theta \theta}$ ); however it impacts the number of equations computed for distinguishability. The worst possible ordering results in the computation of all the Taylor coefficients associated with the order of the expansion that yields a contradiction in (10). This expansion's order is labeled $k^{*}$ and the number of equations corresponding to the worst ordering is given as follows:

$$
v_{\max }=\frac{\left(k^{*}+m\right) !}{k^{*} ! m !}+\beta .
$$

Table 2 gives the lower bound as well as the actual number of equations needed to test for the distinguishability of Examples 1 and 4. A second-order expansion and first-order expansion were needed for Examples 1 and 4, respectively.

\section{CONCLUSIONS}

This paper has presented a method for determining the distinguishability and identifiability of smooth non-linear algebraic models describing contact states. Just as contact state estimation is a dual problem involving the estimation of both model parameters as well as contact states, the Taylor series method provides a unified approach to testing the capability of candidate models to estimate both the parameters and the states. The complexity of the method depends on the number of Taylor coefficients that need to be computed. For kinematic pose equations, it can be shown that this number is bounded above and below for identifiability testing and lower bounded for distinguishability testing. For the examples considered, a modest number of terms were needed for testing.

In contrast to on-line, numerical methods, the Taylor series approach is an analytical method that replaces local results based on the sensor variable path with results which are global with respect to the space of sensor variables. Consequently, it can be used as a tool to select appropriate contact models and sensors in the design of a contact state estimator. Furthermore, as demonstrated by the last example, the 
method can be used to determine appropriate constraints under which given models will be valid for contact state estimation.

While the examples presented here involved only elemental contacts based on pose equations, the methodology is equally applicable to more complex contacts using additional sensor modalities. In particular, force and velocity measurements can be utilized to model multiple contacts as non-linear homogeneous equations using wrench and twist reciprocity properties.

\section{Acknowledgements}

The support of the National Science Foundation under grant IIS-9988575 is gratefully acknowledged.

\section{REFERENCES}

1. J. De Schutter, H. Bruyninckx, S. Dutre, J. De Geeter, J. Katupitiya, S. Demey and T. Lefebvre, Estimating first order geometric parameters and monitoring contact transitions during force controlled compliant motion, Int. J. Robotics Res. 18 (12), 1161-1184 (1999).

2. T. Debus, P. Dupont and R. Howe, Contact state estimation using multiple model estimation and hidden Markov model, Int. J. Robotics Res. 23 (4-5), 399-413 (2004).

3. B. Eberman, A model-based approach to Cartesian manipulation contact sensing, Int. J. Robotics Res. 16 (4), 508-528 (1997).

4. T. Lefebvre, H. Bruyninckx and J. De Schutter, Polyhedral contact formation modeling and identification for autonomous compliant motion, IEEE Trans. Robotics Automat. 19 (1), 26-41 (2003).

5. K. Schröer, L. Uhl, S. Albright and M. Huttenhofer, Ensuring solvability and analyzing results of the non-linear robot calibration problem, in: Proc. Second Int. Symp. Measurement Control Robotics, pp. 851-858 (1992).

6. S. Hirai and I. Kazuaki, Recognition of contact state based on geometric model, in: Proc. IEEE Int. Conf. Robotics Automat., pp. 1507-1512 (1992).

7. J. Rosell, L. Basañez and R. Suarez, Contact identification for robotics assembly tasks with uncertainty, in: 6th IFAC Symp. Robot Control, Vol. 2, pp. 415-420 (2000).

8. J. Xiao and L. Lianzhong, Contact states: representation and recognizability in the presence of uncertainties, in: Proc. IEEE Conf. Intell. Robots Syst. (1998).

9. J. Xiao and L. Zhang, Contact constraint analysis and determination of geometrically valid contact formations from possible contact primitives, IEEE Trans. Robotics Automat. 13 (3), 456466 (1997).

10. M. Gautier and W. Khalil, Direct calculation of minimum set of inertial parameters of serial robots, IEEE Trans. Robotics Automat. 6 (3), 368-373 (1990).

11. An C. Atkeson and J. Hollerbach, Estimation of inertial parameters of manipulator loads and links, Int. J. Robotics Res. 5 (3), 101-119 (1986).

12. S. Y. Sheu and M. Walker, Basis sets for manipulator inertial parameters, in: Proc. IEEE Conf. Robotics Automat., pp. 1517-1522 (1989).

13. B. Eberman, in: Contact sensing: a sequential decision approach to sensing manipulation contact features, PhD Thesis, MIT, Artificial Intelligence Laboratory, pp. 155-158 (1995).

14. L. Ljung and T. Glad, On global identifiability for arbitrary model parametrizations, Automatica 30 (2), 265-276 (1994). 
15. M. Chapell, K. Godfrey and S. Vajda, Global identifiability of non-linear systems with specified inputs: a comparison of methods, Math. Biosci. 102, 41-73 (1990).

16. A. Walter, Y. Lecourtier and A. Raksanyi, On the distinguishability of parametric models with different structures, in: Mathematics and Computers in Biomedical Applications, IMACS, J. Eisenfeld and C. DeLisi (Eds), pp. 145-160. Elsevier Science, Amsterdam (1985).

17. A. Raksanyi, Y. Lecourtier and E. Walter, Identifiability and distinguishability testing via computer algebra, Math. Biosci. 77, 245-266 (1985).

18. Y. Lecourtier, E. Walter and P. Bertrand, Identifiability testing for state-space models, in: 6th IFAC Symp. Identification Syst. Parameter Estimation, pp. 794-799 (1982).

19. E. Walter, Y. Lecourtier and J. Happel, On the structural output distinguishability of parametric models, and its relations with structural identifiability, IEEE Trans. Automat. Control 29 (1) 56-57 (1984).

20. E. Walter and L. Pronzato, On the identifiability and distinguishability of non-linear parametric models, Math. Comput. Simul. 42, 125-134 (1996).

21. S. Vajda, K. R. Godfrey and H. Rabitz, Similarity transformation approach to identifiability analysis of non-linear compartmental models, Math. Biosci. 93, 217-248 (1989).

22. M. S. Grewal and K. Glover, Identifiability of linear and non-linear dynamical systems, $\underline{I E E E}$ Trans. Automat. Control 21, 833-837 (1976).

23. H. Pohjanpalo, System identifiability based on the power series expansion of the solution, Math. Biosci. 41, 21-33 (1978).

24. T. Debus and P. Dupont, Distinguishability and identifiability of contact states, in: Proc. IEEE Int. Conf. Robotics Automat., pp. 1135-1140 (2004).

25. A. O. Farahat, B. S. Graves and J. C. Trinkle, Identifying contact formations in the presence of uncertainty, in: Proc. IEEE Int. Conf. Intell. Robots Syst., pp. 59-64 (1995).

\section{ABOUT THE AUTHORS}

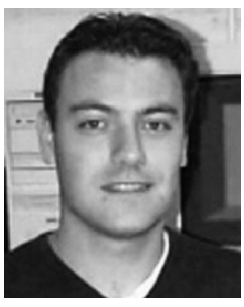

Thomas J. Debus received a BS degree in mechanical engineering from the University of Versailles, France, in 1995, and an MS degree in mechanical engineering in 2000 from Boston University, Boston, USA, where he is currently pursuing a $\mathrm{PhD}$ degree.

His research interests include the planning and analysis of robotics tasks in unstructured environments, teleoperated systems and man-machine cooperation.

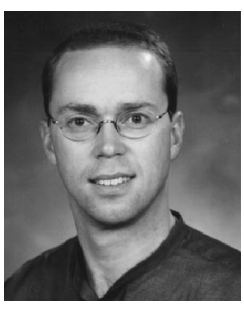

Pierre E. Dupont received the BS, MS and PhD degrees in Mechanical Engineering from Rensselaer Polytechnic Institute in 1982, 1984 and 1988, respectively. He was a postdoctoral fellow with the Center for Intelligent Control Systems at Harvard University from 1988 to 1990 . He joined Boston University in 1990 where he is currently an Associate Professor in the Department of Aerospace and Mechanical Engineering. His research interests include robotics, control systems and medical applications. He is an Associate Editor of the IEEE Transactions on Robotics, a senior member of the IEEE and a member of the ASME. 


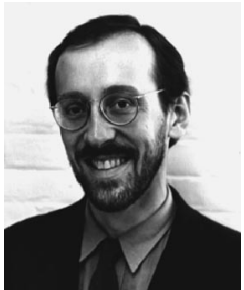

Robert D. Howe received a BA in physics from Reed College, then worked as a design engineer in the electronics industry. He attended graduate school at Stanford University and received a $\mathrm{PhD}$ in mechanical engineering in 1990 . He then joined the faculty of the Division of Engineering and Applied Sciences at Harvard University, where he is currently Gordon McKay Professor of Engineering. Dr. Howe received the National Science Foundation Young Investigator Award in 1993 and the Whitaker Foundation Biomedical Engineering Research Grant (career development award) in 1995.

Dr. Howe's research interests focus on robot and human hands and the sense of touch. He has developed new tactile sensors for robot fingers, and demonstrated novel control algorithms based on these tactile sensor signals. His biomechanics research projects involve measurement of the mechanical impedance of human hands and modeling of mechanoreceptor neural function. Biomedical applications of this work include the development of new technology for minimally invasive surgery. 